\title{
Digital spectrometer for prompt fission neutron spectrum measurements
}

\author{
Pavel Prusachenko a , Ivan Bondarenko, Vladimir Ketlerov, Vitaly Khryachkov, and Vladislav Poryvaev \\ Institute for Physics and Power Engineering, Obninsk, Russian Federation
}

\begin{abstract}
This paper presents a digital neutron spectrometer based on simultaneous digitizing of the signals from the fission chamber and a scintillation detector. The scintillation detector is based on stilbene crystal. The intrinsic detection efficiency of the used stilbene crystal and the energy dependence of the light output for the recoil protons were measured. It is shown, that the method allowed us to achieve time resolution of $1.5 \mathrm{~ns}$ and an $\operatorname{good} \mathrm{n} / \gamma$ separation down to neutron energies of $400 \mathrm{keV}$.
\end{abstract}

\section{Introduction}

Prompt neutron spectra are very important for criticality calculations of nuclear reactors. The most accurate method for measuring the neutron spectra is the time-of- flight method. Organic scintillators are the most frequently used as a neutron detector. Unfortunately, they have considerable sensitivity to gamma rays accompanying fission process. Good separation of events caused by neutrons and gamma quanta is an actual problem, especially for low energy particles.

An important advantage of digital signal processing is the possibility to analyze the pulse shape in detail. This makes it possible to optimize the background conditions, significantly improve a time resolution and achieve a higher peak load $[1,2]$.

This paper presents a spectrometer based on simultaneous digitizing the signal from the fission chamber and a scintillation detector (with organic scintillator). A correlation analysis method was used for the processing of digitized signals. It is shown, that the method allows achieving the time resolution about $1.5 \mathrm{~ns}$ and the $\operatorname{good} \mathrm{n} / \gamma$ separation down to neutron energies of $400 \mathrm{keV}$.

\section{Experimental setup}

Developed digital spectrometer consists of a fission chamber, a scintillation detector (based on organic scintillator), a detector shielding and a dual-channel waveform digitizer. Experimental setup is shown in Fig. 1.

The ${ }^{252} \mathrm{Cf}$ layer, with strength of about 67000 fission fragments per second, was located on the fission chamber cathode. Flight path in the experiment was 2 meters. The neutron detector was stilbene crystal with a diameter of $3 \mathrm{~cm}$ and a thickness of $5 \mathrm{~mm}$ in combination with a photomultiplier ET Enterprise 9813QB.

A specialized neutron shielding was used during the measurements. It was a cone filled with the mixture of

\footnotetext{
a e-mail: prusachenko.pavel@gmail.com
}

paraffin and $\mathrm{LiH}$. The internal channel for neutron detector was shielded by $8 \mathrm{~cm}$ of lead.

A two-channel waveform digitizer Ultraview AD14500 was used in this work. The parameters are: a sampling rate of $500 \mathrm{MHz}$, a bandwidth of $350 \mathrm{MHz}$ and a resolution of 14 bits. Simultaneous digitization of both signals made possible to carry out not only the analysis of form and an amplitude distributions of signals, but also to conduct studying the time characteristics of the signals received by different inputs. The neutron channel signals above the threshold were used as the trigger. Figure 2 shows an example of a single event.

\section{Signal processing}

At the first stage response signal of scintillation spectrometer for electrons was determined. This work was done using standard gamma source ${ }^{60} \mathrm{Co}$. The response signal (shown as the blue line in Fig. 3) was obtained by averaging approximately 5000 individual signals. The resulting signal was used for further processing to determine the type of the detected particle and the time of its occurrence by correlation analysis of the similarity in the shape of the processed signal and the swatch signal.

The amplitude of the correlation peak for the signals caused by gamma quanta is greater than for the signals from neutrons due to the difference in their form. Furthermore, the position of the correlation peak allows us to find the true time of occurrence of the processed signal.

In this work, during the analysis of the array of all received oscillograms, three main types of signals from the neutron detector were identified - the signals from the recoil protons (red line in Fig. 3), electrons (blue line) and the signals produced by the luminescence of quartz input window PMT during deceleration of fast electrons in it (black line). The signal from the recoil protons corresponds to the proton kinetic energy $1.7 \mathrm{MeV}$. These signals are normalized to their area.

Neutron detector calibration was performed using ${ }^{60} \mathrm{Co}$ and ${ }^{137} \mathrm{Cs}$ sources by the right edge of the Compton electrons distributions for corresponding gamma-quanta. 


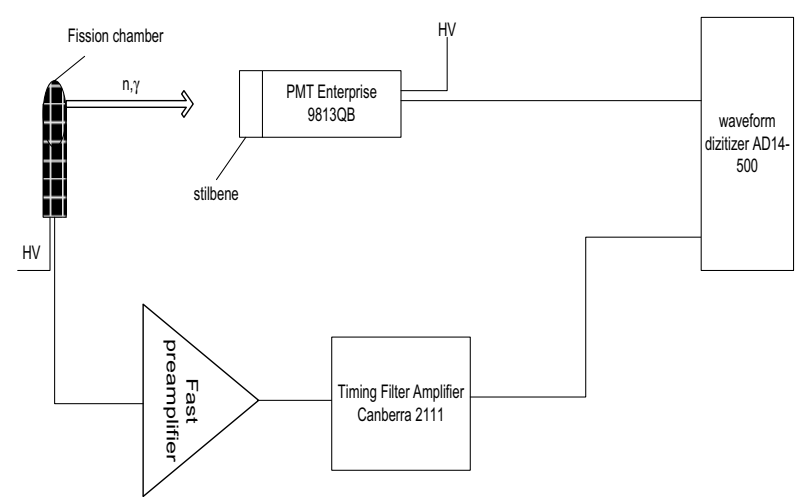

Figure 1. Electronic scheme of experimental setup.

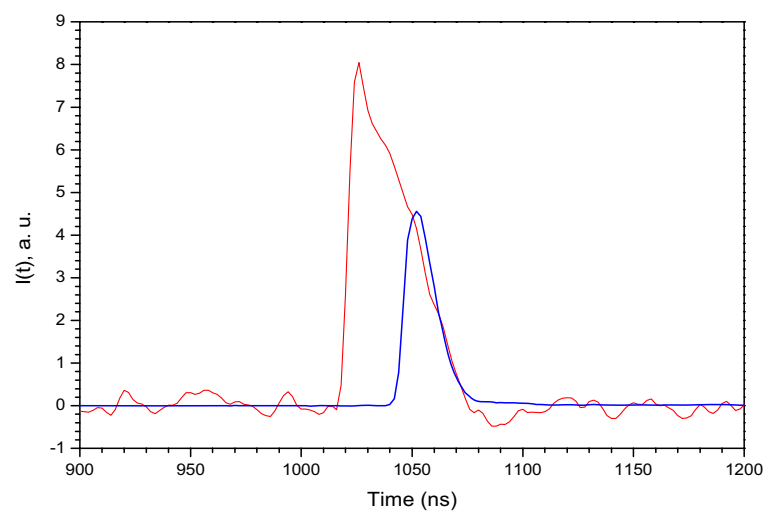

Figure 2. An example of a single event, which contains oscillograms from fission chamber (red) and neutron detector (blue).

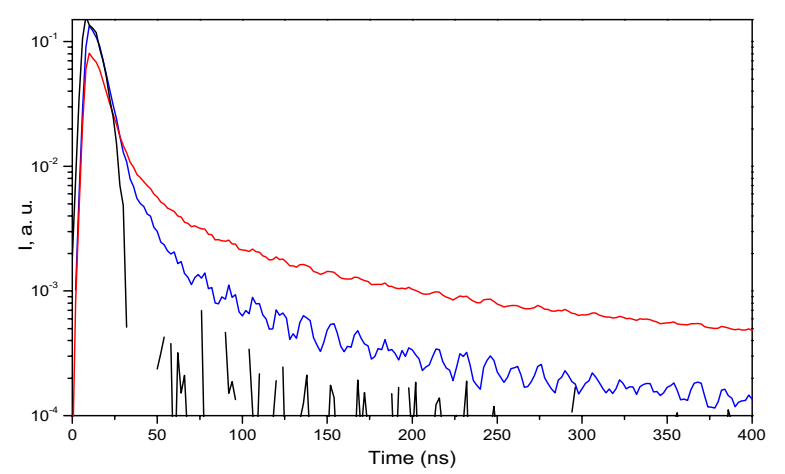

Figure 3. The response of the neutron detector on electrons, recoil protons and outbreaks in a quartz input window.

\section{Result and discussion}

\subsection{Separation quality}

Figure 4 shows the separation quality for the method of correlation analysis. The separation is made in the parameter $\mathrm{R}$, equal to the ratio of the maximum of correlation peak (MCP) to the area of signal (A) (Eq. (1)).

$$
R=\frac{M C P}{A}
$$

The estimation of the separation quality in the work served as a parameter Figure of Merit (FoM), which is a ratio of

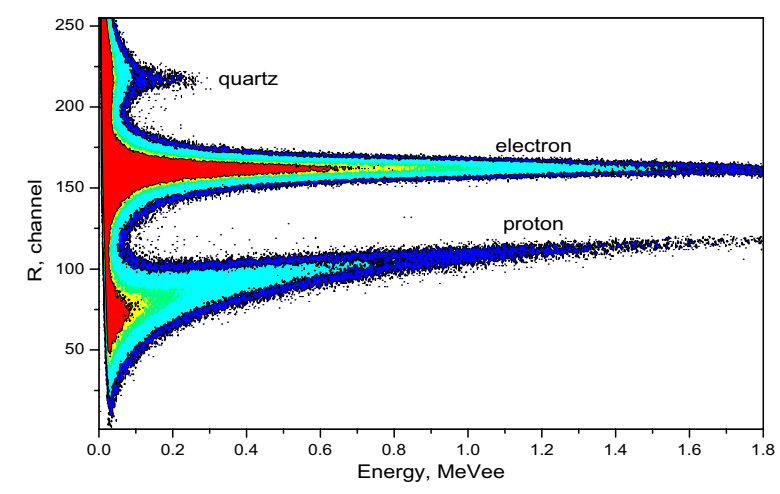

Figure 4. The two-dimensional spectrum in the axes of parameter $\mathrm{R}$ - energy in MeVee.
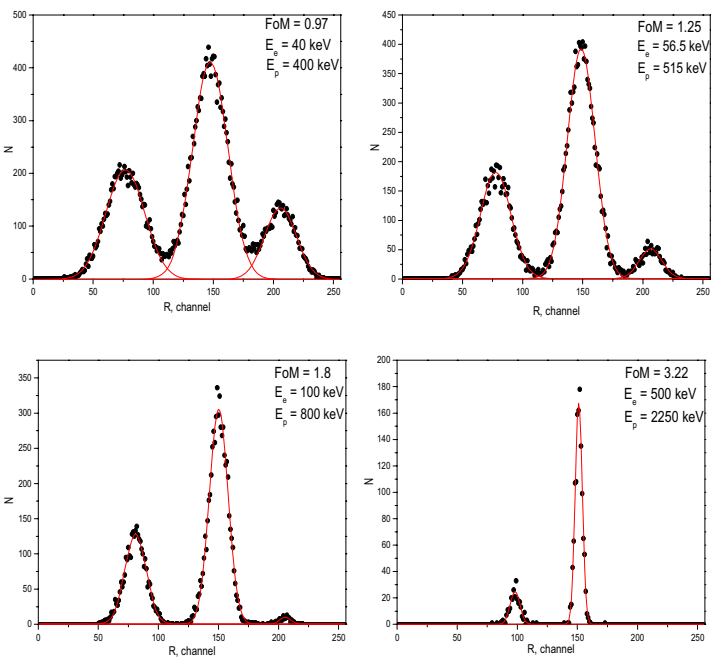

Figure 5. Slices of the two-dimensional spectrum (Fig. 4) at different energies. In top right corner of each picture values of FoM parameter, energy of protons and electrons are given.

the difference of the first moments of the distributions to the sum of the full widths at half-height (Eq. (2)).

$$
F o M=\frac{M_{\gamma}+M_{n}}{F W H M_{\gamma}+F W H M_{n}}
$$

Figure 5 shows slices of two-dimensional spectrum at different energies and their fitting by Gaussians. It is shown that separation quality increases with energy increasing. Processing method used in this work allowed us to identify confidently the pulses of electrons and protons at energy $40 \mathrm{keVee}$, which corresponds to a neutron energy of $400 \mathrm{keV}$ (see Sect. 4.3).

\subsection{Time-of-flight spectra}

The two-dimensional spectrum is shown in the Fig. 6. On the axes - the time of flight of the particle and separation parameter R (Eq. (1)), produced using ${ }^{252} \mathrm{Cf}$ source. The figure clearly shows three well-localized event group corresponding neutrons (the lower part of the spectrum), the gamma quanta (the middle part of the spectrum), and the events corresponding to the luminescence of quartz input window of photomultiplier (upper part of the spectrum). The numbers in Fig. 6 are showing region of neutrons (1) and region of background associated with gamma quanta from inelastic scattering of fast neutrons on the detector material and structural materials. 


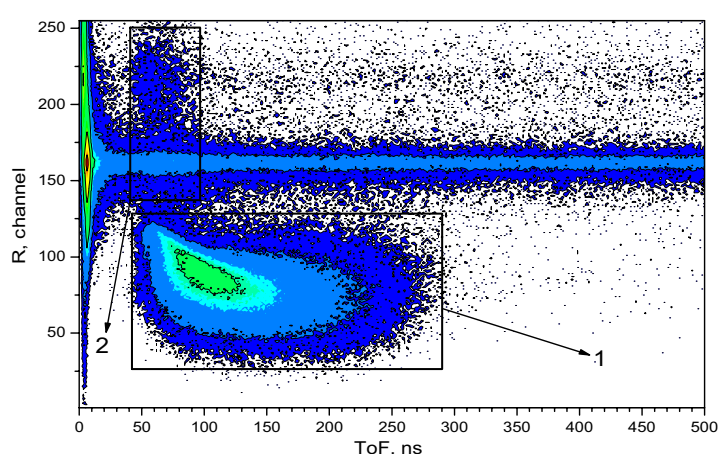

Figure 6. The two-dimensional spectrum with the axes: time-offlight and the value of separation parameter $\mathrm{R}$.

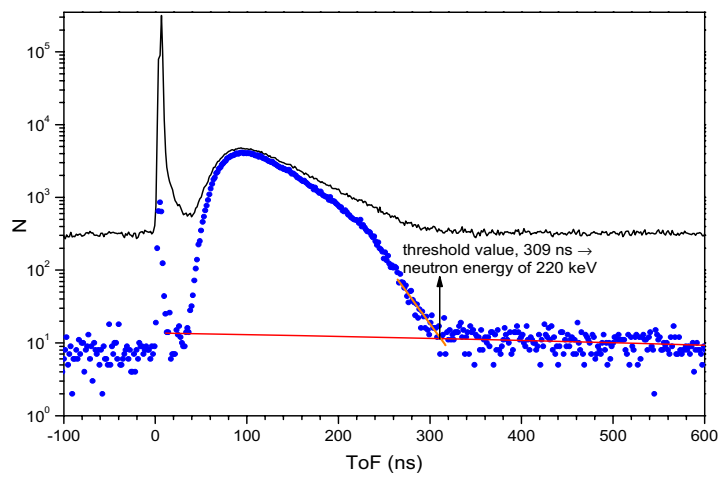

Figure 7. Integral TOF spectrum (black line) and the neutron time of flight spectrum (point) after the suppression of background gamma rays. Red line - background.

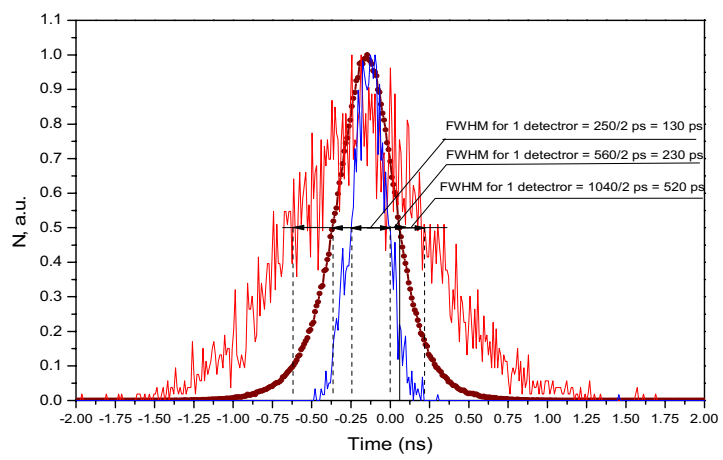

Figure 8. The time resolution, a total for two neutron detectors obtained by coincidence on ${ }^{60} \mathrm{Co}$.

Partial one-dimensional time-of-flight spectrum (Fig. 7) can be obtained from the two-dimensional timeof-flight spectrum by setting the appropriate window to the parameter $\mathrm{R}$. The reached degree of suppression background of gamma-quanta is 350. It is clear from Fig. 7 that TOF spectrum takes the background values at the time of flight of $309 \mathrm{~ns}$. In the experiment described, the time of flight $309 \mathrm{~ns}$ corresponds to neutron energy of $220 \mathrm{keV}$ (for the flight path of $2 \mathrm{~m}$ ). It can be concluded that the threshold of neutrons detection in this experiment was $220 \mathrm{keV}$.

Time resolution in the work was estimated by the peak width of the prompt gamma rays. The peak width at half maximum was $1.5 \mathrm{~ns}$ throughout the range above the threshold. This time resolution makes measurements in the field of high-energy neutrons up to $13 \mathrm{MeV}$ possible. The time resolution of used stilbene crystal in combination with

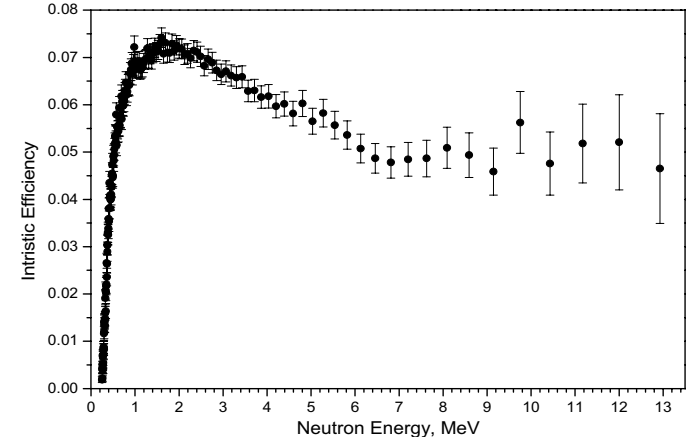

Figure 9. Intrinsic detection efficiency for stilbene crystal used in the work.

a photomultiplier ET Enterprise 9813QB was measured by using a coincidence on ${ }^{60}$ Co source (Fig. 8). It was found that a time resolution for a neutron detector was $230 \pm 1 \mathrm{ps}$ (wine point on Fig. 8) in the energy range $36-1117 \mathrm{keV}$. For the area of the first Compton edge $(969 \mathrm{keV}$, blue line on Fig. 8) time resolution was $129 \pm 5$ ps. For the region less than $100 \mathrm{keV}(36-74 \mathrm{keV}$, red line on Fig. 8) the time resolution was $520 \pm 30 \mathrm{ps}$. The results mean that fission chamber gives the main contribution to the width of the peak of prompt gamma quanta. The time characteristics of the spectrometer can be significantly improved by improving its design.

\subsection{Efficiency of neutron detector}

The intrinsic detection efficiency of neutron detector has been obtained on the basis of the standard spectrum of ${ }^{252} \mathrm{Cf}$ prompt fission neutrons taken from ENDF-BVII.1 library and the spectrum obtained experimentally [3,4]. The obtained results are shown in Fig. 9. The statistical error, uncertainty of the standard spectrum from ENDFBVII, the uncertainty of determining the flight path $( \pm 2.5 \mathrm{~mm})$ were taken into account in final error estimation. The biggest errors were for energies above $9 \mathrm{MeV}$ and below $0.5 \mathrm{MeV}$.

A correction on the width of a bin (Eq. (3)) was applied for the correct image of the received data, as pointed on the graph. This adjustment must be done to areas of rapid change in the spectrum, since in this areas average energy bin is shifted. This adjustment was made on the basis of the expression proposed in [5] and used in the [6].

$$
E_{b}=T \ln \frac{E_{2}-E_{1}}{T\left(e^{-\frac{E_{1}}{T}}-e^{-\frac{E_{2}}{T}}\right)}
$$

Here $E_{1}$ and $E_{2}$ - boundary energy of bin, $T$ - the decay constant, the resulting description of the areas of spectrum exponential change.

\subsection{Light output for the recoil proton for stilbene crystal}

For organic scintillators there is dependence of the total light output from the particle linear energy losses. Accordingly, for electrons, whose linear energy losses are practically independent on the energy, the light output will increase linearly with increasing of particle energy. Situation is different for heavy charged particles. The 


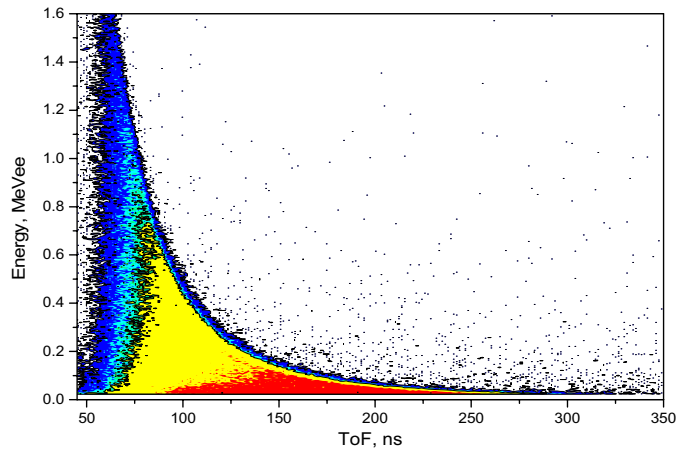

Figure 10. The two-dimensional spectrum in the axes of the timeof-flight - light output for calibration by electrons.

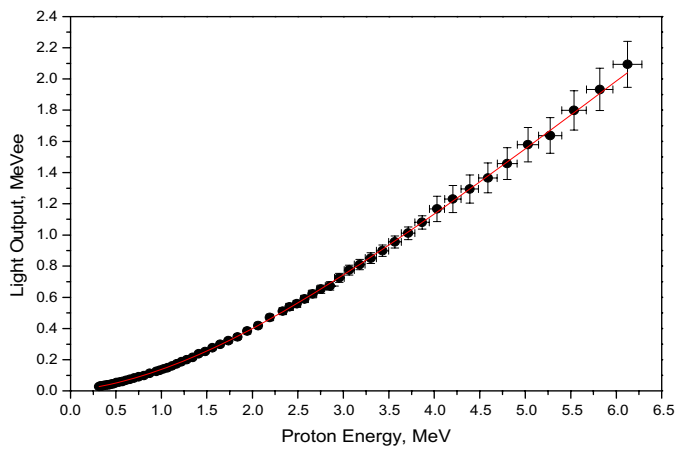

Figure 11. Light output for recoil protons. Points - experiment, line - an empirical description by Eq. (4).

linear energy losses are strongly dependent on the kinetic energy and particle type [7].

The basic process that contributes to the detection of neutrons in an organic scintillator is the process of elastic scattering of neutrons in the nuclei of hydrogen. Therefore, it is very important to know the dependence of the light output on the energy of the recoil proton.

In this work, the energy dependence of the light output of protons for stilbene crystal was experimentally measured. Figure 10 shows a two-dimensional spectrum with the axes of the time-of-flight - the energy deposited in the detector, after the removal of the background of the gamma quanta. Each slice on the time of flight for the twodimensional spectrum is the spectrum of the recoil protons for fixed neutron energy. The edge of this distribution corresponds to the energy of the neutrons. Therefore, using the calibration constants obtained for the electrons the dependence of the light output for the recoil protons can be restored (Fig. 11, a point).

There are several approaches to the empirical description of the energy dependence of the light output for heavy charged particles [7-9]. The best way for description of the data obtained in this study was the method proposed Cecil (Eq. (4)) in paper [9]. The result of empirical description is presented in Fig. 11 (solid line).

$$
L(M e V e e)=a \cdot E_{p}-b \cdot\left(1-e^{-c E_{p}^{d}}\right)
$$

Here $\mathrm{a}=0.45 \pm 0.01, \mathrm{~b}=0.76 \pm 0.06, \mathrm{c}=0.54 \pm 0.03$, $\mathrm{d}=1.032 \pm 0.08$.

\section{Conclusions}

Fully digital neutron spectrometer based on the stilbene crystal, the parallel plate ionization chamber and the waveform digitizer was developed. The intrinsic detection efficiency was obtained. It was shown that the method of correlation analysis of signals achieves excellent $n / \gamma$ separation down to energy of $40 \mathrm{keVee}$, corresponding to energy of $400 \mathrm{keV}$ of recoil protons. Possibility of neutron and gamma-quanta separation down to neutron energies of $220 \mathrm{keV}$ was shown. The influence of luminescence of quartz input window of the PMT was taken into account. The time resolution measured by the peak width of the prompt gamma rays from ${ }^{252} \mathrm{Cf}$ was $1.5 \mathrm{~ns}$. The time resolution for a neutron detector was $230 \pm 1 \mathrm{ps}$ in the energy range $36-1117 \mathrm{keV}$. With energy increasing, it improves from $520 \mathrm{ps}$ to $129 \mathrm{ps}$. This makes it possible to analyze the shape of the neutron spectrum in detail. The energy dependence of the light output for the recoil protons was measured. Developed spectrometer can be used for a wide range of tasks related to the measurement of the neutron spectra, both fundamental and applied.

\section{References}

[1] N.V. Kornilov, et al., Nuclear Instruments and Methods A 497, 467 (2003)

[2] D. Cester, et al., Nuclear Instruments and Methods A 748, 33 (2014)

[3] N.V. Kornilov, et al., Annals of Nuclear Energy 27, 1643 (2000)

[4] ENDF-BVII.1 library

[5] D.G. Madland and J.R. Nix, Nucl. Sci. Eng. 81, 213 (1982)

[6] P. Staples, et al., Nuclear Physics A 591, 41 (1995)

[7] J. Iwanowska, et al., Nuclear Instruments and Methods A 781, 44 (2015)

[8] N.V. Kornilov, et al., Nuclear Instruments and Methods A 599, 226 (2009)

[9] R.A. Cecil, et al., Nuclear Instruments and Methods A 161, 439 (1979) 